\title{
MAP2K6 is associated with radiation resistance and adverse prognosis for locally advanced nasopharyngeal carcinoma patients
}

This article was published in the following Dove Press journal: Cancer Management and Research

\author{
Zhanzhan Li \\ $\mathrm{Na} \mathrm{Li}$ \\ Liangfang Shen \\ Department of Oncology, Xiangya \\ Hospital, Central South University, \\ Changsha, Hunan Province 410008, \\ China
}

Correspondence: Liangfang Shen Department of Oncology, Xiangya Hospital, Central South University, No. 87, Xiangya Road, Kaifu District, Changsha, Hunan Province 410008, China Tel/fax +86 73I 89753332

Email liangfangshen86@126.com
Background: Although radiotherapy is the primary therapeutic option for nasopharyngeal carcinoma (NPC), local recurrence and distant metastasis caused by radioresistance are still the major barriers for some NPC patients who cannot benefit from radiotherapy. In this study, we analyzed the association between MAP2K6 expression and radioresistance in patients with locally advanced NPC.

Methods: We collected 120 NPC patients who received radiotherapy in the Xiangya Hospital of Central South University from August 2008 to July 2012. The clinical data and tissue samples of patients were collected. Detection of MAP2K6 was performed using immunohistochemical staining.

Results: The rates of two groups were $19.4 \%$ and $4.2 \%$, and significant difference was observed between MAP2K6 high expression group and low expression group $\left(\chi^{2}=5.817, P=0.016\right)$. The Kaplan-Meier analysis suggested a significant difference in the survival rate between two groups $(P<0.05)$. The results from multivariate Cox regression indicated that the MAP2K6 was independently related to adverse prognosis in $\mathrm{NCP}$ patients $(\mathrm{HR}=3.40,95 \% \mathrm{CI}=1.13-10.26, P=0.030)$. Conclusion: The present study indicated that MAP2K6 was correlated with radioresistance, and the elevated expression of MAP2K6 predicted poor prognosis in NPC patients. MAP2K6 may be a new therapy target for radioresistance of NPC.

Keywords: Mitogen-activated protein kinase 6, progression free-survival, radioresistance

\section{Introduction}

Nasopharyngeal carcinoma (NPC) is one of the most prevalent cancers in Southern China and Southeast Asia, which seriously affects the local people's health. ${ }^{1}$ Radiotherapy is the prior selection for NPC therapy. In the past 10 years, radiation therapy technology has been developing by leaps and bounds with the intervention of electronic computers. The application of intensity-modulated radiation therapy (IMRT) has made the curative effect of NPC radiotherapy continue to improve. ${ }^{2-4}$ However, there are still some patients who cannot benefit from this therapy. The local recurrence and distant metastasis caused by radioresistance become the main obstacles for the patients with NPC to obtain satisfactory efficacy. Blindly increasing the dose of radiotherapy can only increase the local control rate and reduce the distant metastasis rate, but it will also increase the radiation damage of the surrounding normal tissues and organs such as the brain stem and spinal cord and even result in serious short-term and long-term adverse reactions. ${ }^{5}$ It was reported that 5-year disease-free survival rate of NPC patients with stages I-IVB was $83.3 \% .{ }^{6}$ However, $\sim 20 \%$ of NPC patients have local recurrence and 
distant metastasis. ${ }^{7}$ Previous studies had preliminarily identified the risk factors and possible mechanisms of NPC, but the specific mechanism of local recurrence and metastasis caused by radiotherapy resistance is not yet clear. ${ }^{8}$ Therefore, it will provide new potential targets for radiotherapy sensitization treatment of NPC via exploring molecular mechanisms and key regulating factors of radiotherapy resistance.

Protein kinases belong to a class of enzymes that catalyze phosphorylation of proteins, and they can transfer gammaphosphate from adenosine triphosphate (usually ATP) to serine, threonine, or tyrosine residues by phosphorylation. Approximately $2 \%$ of eukaryotic genes are protein kinases, and there are $>500$ kinases in the mammalian genome. Protein kinases, such as receptor tyrosine kinases, cyclindependent kinases, and MAP kinases (MAPKs) accounts for the majority of all kinases, and they play crucial roles in cellular metabolism, cell signaling, protein regulation, cell transport, cell division, and many other forms of cancer cell pathways. ${ }^{9}$ Mitogen-activated protein kinase kinase 6 (MAP2K6) is an upstream kinase of the p38/MAPK signal pathway. ${ }^{10}$ MAP2K6 is involved in various physiological and pathological processes such as cell growth, development, division, and inflammatory reactions. ${ }^{11}$ In recent years, some studies have found that MAP2K6 may be associated with the occurrence and progression of tumors. In fact, $20 \%$ of human cancers are related to the occurrence of MAPK. Parray et $\mathrm{al}^{12}$ use immune fluorescence technology to systematically evaluate the MAP2K6 protein expression of different types of human tumor samples. The results suggested that MAP2K6 expression was significantly increased in esophageal cancer, gastric cancer, and colon cancer compared with the control, and the high expression of MAP2K6 was also found in the kidney, intestine, and lung cancer primary tissues. These results showed that MAP2K6 was probably involved in the occurrence and progression of human tumors and could be potentially treated as a new diagnostic or prognostic biomarker for cancers. In this study, we explored the association between MAP2K6 and radioresistance in patients with locally advanced NPC.

\section{Methods}

\section{Patients and tissue samples}

We retrospectively selected 120 NPC patients from the Department of Radiation, Xiangya Hospital of Central South University, between August 2008 and July 2012. The clinical data and paraffin section samples were collected according to the following the criteria: 1) all patients were confirmed by pathology examination; 2) no distant metastasis and other tumors when patients were confirmed; 3) paraffin-embedded tissue samples were available for immunohistochemistry analysis; and 4) patients did not receive other anticancer treatment before chemoradiotherapy or surgery treatment after radiotherapy. TNM staging referred to American Joint Committee on Cancer (AJCC) staging system for NPC. The follow-up ended on May 31, 2016. The primary outcome was recurrent or metastatic. Progression-free survival (PFS) time was defined from therapy to recurrence and metastasis. All outcomes must be confirmed by clinical examination. NPC patients were divided into radioresistance group and radiosensitive group. ${ }^{13}$ This study was approved by the ethics committee of the Xiangya Hospital Central South University, and all patients provided written informed consent, and this study was conducted in accordance with the Declaration of Helsinki.

All patients received external beam IMRT. IMRT was applied as follows: 68-74 Gy/30-33 fraction to planned targeted volume (PTV) $\mathrm{nx}, 68-70 \mathrm{~Gy} / 30-33$ fraction to PTVnd, 60-64 Gy/30-33 fraction to PTV1, and 50-56 Gy/30-33 fraction to PTV2, and received two periods of induction chemotherapy with docetaxel $\left(65 \mathrm{mg} / \mathrm{m}^{2} / \mathrm{d}_{1}\right)$ plus dedaplatin (NDP; $80 \mathrm{mg} / \mathrm{m}^{2} / \mathrm{d}_{1}$ ) every 3 weeks, followed by six cycles of concurrent chemotherapy with NDP (40 mg/ $\mathrm{m}^{2} / \mathrm{d}_{1}$ ) every week. Besides, some patients received sodium glycididazole through intravenous $750 \mathrm{mg} / \mathrm{m}^{2} /$ fraction, 3-5 fraction/week, within 1 or 2 hours before radiotherapy. Some patients received nimotuzumab (100-200 mg/week) and cetuximab (400 mg/m²/week).

\section{Immunohistochemical staining}

Immunostaining detection was conducted under the guidelines of ChemMate kit (Dako Denmark A/S, Glostrup, Denmark) and 3,3'-diaminobenzidine as the chromogen, which has been described in the previous studies. ${ }^{14}$ First, paraffin section was dewaxed, sealed by $3 \% \mathrm{H}_{2} \mathrm{O}_{2}$ for 10 minutes, and followed by distilled water wash for 5 minutes in the shake bed. Second, slice was placed in heat-resistant glass containers with sodium citrate buffer, $92^{\circ} \mathrm{C}-95^{\circ} \mathrm{C}, 5$ minutes; remove the residue and replenish preheated sodium decanoate buffer; $92^{\circ} \mathrm{C}-95^{\circ} \mathrm{C}, 5$ minutes. Third, specimens were incubated overnight at $4^{\circ} \mathrm{C}$ with the anti-MAP2K6 antibody at a dilution of 1:400 (Cell Signaling Technology, Inc., Beverly, MA, USA). Detection of immunostaining was performed using the ChemMate kit (Dako Denmark A/S) 
with 3,3'-diaminobenzidine as the chromogen. The nonimmune isotype antibodies replaced the primary antibody as the negative control.

The evaluation of staining was conducted by two experienced pathologists. The pathologists scanned the tissue specimens under the low-power $(\times 40)$ and high-power $(\times 400)$ magnification. The positive and negative results were according to the staining intensity and scope. The criteria were as follows: sample with no positive cells were negative $(-),<25 \%$ of cells: weakly positive $( \pm), 25 \%-50 \%$ : positive $(+),>50 \%$ of cells: strongly positive $(++) .{ }^{15}$ We defined the negative or weak positive as the MAP2K6 low expression group and the positive or strong positive as MAP2K high expression group.

\section{Statistical analyses}

First, we divided NPC patients into radioresistant and radiosensitive groups. The clinical parameters between low and high expression groups were compared by using chi-squared test for the qualitative data (age: $\geq 45$ vs 45 years; gender: male vs female; radiotherapy: $\geq 73.27$ vs $<73.27$ Gy; AJCC: stage IVa vs III; chemotherapy: no vs yes; targeted therapy: no vs yes; sensitization therapy: no vs yes) and Student's $t$-test for the quantitative data. Second, NPC patients were divided into low expression group and high expression of MAP2K6. The chi-squared and Student's $t$-test were also used for comparison of clinical feature between two groups. HR with $95 \%$ CIs was calculated for assessing the relationship between MAP2K6 and prognosis through univariate and multivariate Cox regression analysis. The survival curves of MAP2K6 low and high expression was plotted by Kaplan-Meier analysis. We performed the whole analyses by using SPSS 20.0 (IBM Corporation, Armonk, NY, USA) and GraphPad Prism 6.0 (GraphPad Software, Inc., La Jolla, CA, USA). $P<0.05$ was considered to be statistically significant.

\section{Results}

\section{General characteristics of NPC patients}

One hundred and twenty NPC patients were included in the follow-up, including 16 radioresistance patients and 104 radiation-sensitive patients, including 52 females and 68 males. The median age was 47 years, ranging from 28 to 70 years. The follow-up duration was from 12 to 71.8 months, with the median of 42 months. There were 52 patients younger than 45 years and 68 patients aged 45 years or older. Fifty-two patients were classified as stage III and 68 patients as stage IVa. Eleven patients did not receive chemotherapy. Sixty-six patients receive sensitization therapy before radiotherapy. Fourteen patients took nimotuzumab. Clinical and histopathological data of NPC patients are presented in Table 1. The MAP2K6 expression level in radioresistant group was significantly higher than that in the radiosensitive group $(P=0.016)$. There were no significant differences in age $(P=0.971)$, gender ratio $(P=0.263)$, radiotherapy dose $(P=0.543)$, AJCC stage $(P=0.263)$, chemotherapy $(P=0.620)$, target therapy $(P=0.428)$, and sensitization $(P=0.517)$ between two groups.

We also divided NPC patients into MAP2K6 high expression group and MAP2K6 low expression group according to the MAP2K6 expression level. Clinical characteristics were also compared between two groups. The results are listed in Table 2. No significant differences were observed in clinical parameters between two groups $(P>0.05)$.

\section{MAP2K6 and radiotherapy resistance}

According to the results of immunohistochemical, MAP2K6 was mainly located in the nucleus and cytosol of cell, and the MAP2K6 in the radioresistant group was significantly stronger than in the radiosensitive group (Figure 1). Fourteen patients were confirmed as radioresistance in the MAP2K6 high expression group, and there were two patients in the MAP2K6 low expression group. The rates of two groups were $19.4 \%$ and $4.2 \%$, and significant difference was observed between the high expression group and low expression group $\left(\chi^{2}=5.817, P=0.016\right)$.

\section{MAP2K6 and survival}

The Kaplan-Meier analysis showed a significant difference in the survival curve between the high expression group and low expression group $(P<0.05$, Figure 2$)$. We conducted the univariate and multivariate Cox regression analyses to identify the relationship between MAP2K6 expression and PFS in the NPC patients, including the following parameters: age, gender, therapy dose, chemotherapy, AJCC stage, targeted therapy, sensitization therapy, and MAP2K6 expression. The univariate results showed that MAP2K6 was associated with adverse prognosis in NPC patients $(\mathrm{HR}=3.46,95 \% \mathrm{CI}$ $=1.17-10.22, P=0.025)$. The other variables are presented in Table 3. The multivariate results indicated that the MAP2K6 high expression was independently related to adverse prognosis in NPC patients $(\mathrm{HR}=3.40,95 \% \mathrm{CI}=1.13-10.26$, $P=0.030)$. Other variables were not associated with PFS in NPC patients (Table 4).

\section{Discussion}

The current study qualitatively evaluated the expression of MAP2K6 in NPC tissues via immunohistochemistry, and 
Table I General characteristics of study patients

\begin{tabular}{|c|c|c|c|c|}
\hline \multirow[t]{2}{*}{ Parameters } & \multicolumn{2}{|l|}{ Radiation sensitivity, n (\%) } & \multirow[t]{2}{*}{$\chi^{2}$} & \multirow[t]{2}{*}{$P$-value } \\
\hline & Radiation-resistant group & Radiation-sensitive group & & \\
\hline Age (years) & & & 0.001 & 0.971 \\
\hline$<45$ & $7(13.5)$ & $45(86.5)$ & & \\
\hline$\geq 45$ & $9(13.2)$ & $59(86.8)$ & & \\
\hline Gender & & & 1.254 & 0.263 \\
\hline Female & $9(10.3)$ & $43(82.7)$ & & \\
\hline Male & $7(17.3)$ & $61(89.7)$ & & \\
\hline Radiotherapy dose & & & 0.371 & 0.543 \\
\hline$<73.27$ & $9(15.3)$ & $50(84.7)$ & & \\
\hline$\geq 73.27$ & $7(11.5)$ & $54(88.5)$ & & \\
\hline AJCC stage & & & 1.254 & 0.263 \\
\hline III & $9(17.3)$ & $43(82.7)$ & & \\
\hline $\mathrm{IVa}$ & $7(10.3)$ & $61(89.7)$ & & \\
\hline Chemotherapy & & & 0.246 & 0.620 \\
\hline Yes & $14(12.8)$ & 95 (87.2) & & \\
\hline No & $2(18.2)$ & $9(8 I .8)$ & & \\
\hline Targeted therapy & & & 0.526 & 0.428 \\
\hline Yes & $\mathrm{I}(7.1)$ & $13(92.9)$ & & \\
\hline No & $15(14.2)$ & 91 (85.8) & & \\
\hline Sensitization therapy & & & 0.420 & 0.517 \\
\hline Yes & $10(15.2)$ & $56(84.8)$ & & \\
\hline No & $6(11.1)$ & $48(88.9)$ & & \\
\hline MAP2K6 & & & 5.817 & 0.016 \\
\hline Low & $2(4.2)$ & 46 (95.8) & & \\
\hline High & $14(19.4)$ & $58(80.6)$ & & \\
\hline
\end{tabular}

Abbreviation: AJCC, American Joint Committee on Cancer.

Table 2 Correlation between MAP2K6 expression and clinical pathological features in patients with NPC

\begin{tabular}{|c|c|c|c|c|}
\hline \multirow[t]{2}{*}{ Parameters } & \multicolumn{2}{|c|}{ MAP2K6 expression, n (\%) } & \multirow[t]{2}{*}{$\chi^{2}$} & \multirow[t]{2}{*}{$P$-value } \\
\hline & Low expression & High expression & & \\
\hline Age (years) & & & 0.684 & 0.408 \\
\hline$<45$ & $23(44.2)$ & $29(55.8)$ & & \\
\hline$\geq 45$ & $25(36.8)$ & $43(63.2)$ & & \\
\hline Gender & & & 2.494 & 0.114 \\
\hline Female & $25(66.2)$ & $27(5 \mathrm{I} .9)$ & & \\
\hline Male & $23(33.8)$ & $45(48.1)$ & & \\
\hline Radiotherapy dose & & & 1.801 & 0.179 \\
\hline$<73.27$ & $20(33.9)$ & $39(66.1)$ & & \\
\hline$\geq 73.27$ & $28(45.9)$ & $33(54.1)$ & & \\
\hline AJCC stage & & & 2.042 & 0.153 \\
\hline III & $17(32.7)$ & 35 (67.3) & & \\
\hline $\mathrm{IVa}$ & $31(45.6)$ & $37(54.4)$ & & \\
\hline Chemotherapy & & & 1.505 & 0.220 \\
\hline Yes & $46(42.2)$ & $63(57.8)$ & & \\
\hline No & $2(18.2)$ & $9(8 \mid .8)$ & & \\
\hline Targeted therapy & & & 0.054 & 0.816 \\
\hline Yes & $\mathrm{I}(7.1)$ & $13(92.9)$ & & \\
\hline No & $15(14.2)$ & $91(85.8)$ & & \\
\hline Sensitization therapy & & & 0.275 & 0.600 \\
\hline Yes & $25(37.9)$ & $4 I(62.1)$ & & \\
\hline No & $23(42.6)$ & $31(57.4)$ & & \\
\hline Therapy & & & 5.817 & 0.016 \\
\hline Sensitivity & $46(95.8)$ & \begin{tabular}{|l|}
$58(80.6)$ \\
\end{tabular} & & \\
\hline Resistance & $2(4.2)$ & $14(19.4)$ & & \\
\hline
\end{tabular}

Abbreviations: AJCC, American Joint Committee on Cancer; NPC, nasopharyngeal carcinoma. 

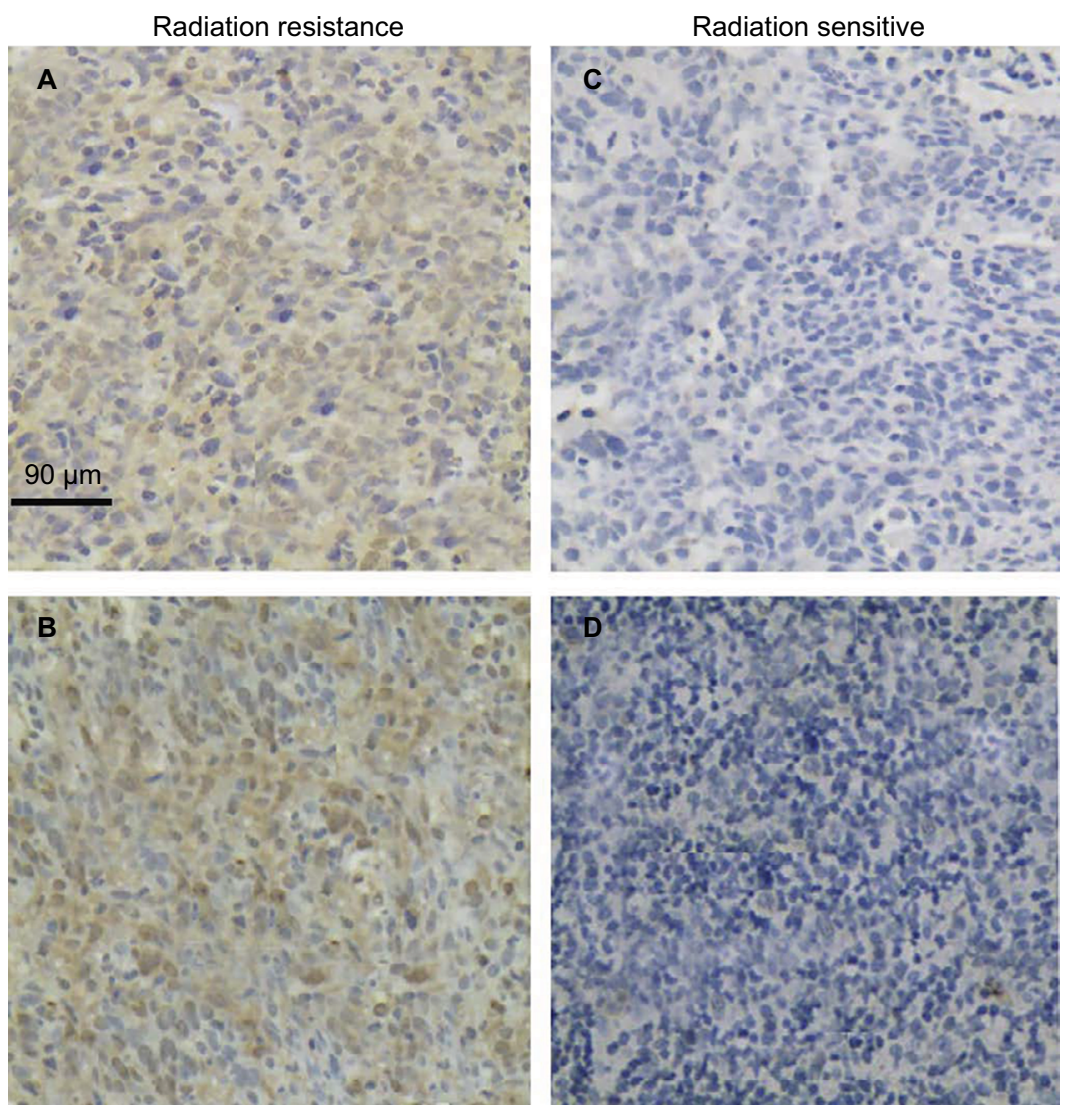

Figure I Expression of MAP2K6 in radiation-resistant (upper) and radiation sensitive (lower) NPC tissues - radiation resistant: (A) 60\% and (B) 0\%; radiation sensitive: (C) $5 \%$ and (D) $10 \%$.

Abbreviation: NPC, nasopharyngeal carcinoma.

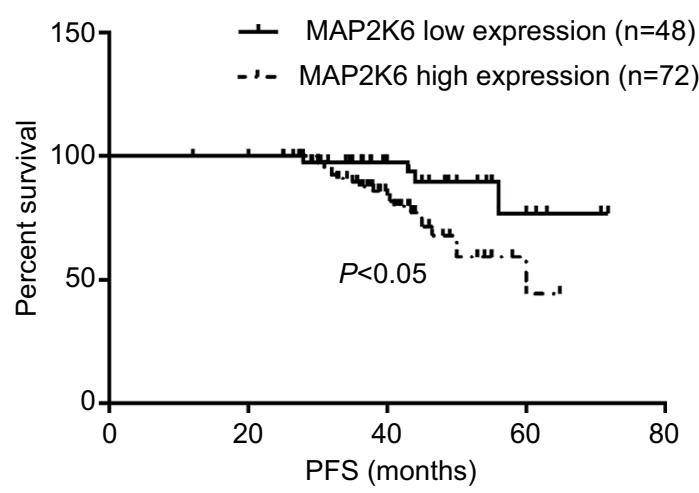

Figure 2 The significant difference in the PFS rate was observed in the patients with low MAP2K 6 expression and high MAP2K 6 expression.

Abbreviation: PFS, progression-free survival.

our results indicated that the expression of MAP2K6 was significantly elevated in the radioresistance NPC tissues. The further analyses indicated that the MAP2K6 expression was associated with the prognosis of NPC patients, and the high expression of MAP2K6 predicted a worse prognosis of patients with NPC.
Although radiotherapy is the primary therapeutic option for NPC, local recurrence and distant metastasis caused by radioresistance are still the major barriers for some NPC patients who cannot benefit from radiotherapy. Therefore, it is significantly important to deeply explore the molecular mechanisms and to identify the key effect factor and related signaling pathways of NPC radioresistance for intervention. Previous studies had reported some radioresistance biomarkers. ${ }^{16-18}$

MAP2K6 belongs to one of the families of dual specificity protein kinase and can activate the MAPK. The MAPK can regulate the extracellular signal pathway and is the overlap points of several signals. ${ }^{19}$ MAP2K6 can phosphorylate and activate the p38/MAPK signal pathway under the status of inflammatory and stress. Previous studies have suggested that MAP2K6 plays important roles in cell cycle regulation, transcription, and cell apotosis. ${ }^{20,21}$ Parray et $\mathrm{al}^{22}$ found that the expression of MAP2K6 was significantly higher in esophageal, stomach, and colon cancers than controls using Western blotting and immunofluorescence assay. Overexpression of MAP2K6 is indicative of its role in human cancers and 
Table 3 Univariate Cox regression analyses of clinical prognosis in patients with NPC

\begin{tabular}{|c|c|c|c|c|c|c|}
\hline Factors & $\beta$ & SE & Wald $\chi^{2}$ & HR & $95 \% \mathrm{Cl}$ & $P$-value \\
\hline Age ( $\geq 45$ vs 45 years) & -0.250 & 0.439 & 0.325 & 0.78 & $0.33-1.84$ & 0.569 \\
\hline Gender (male vs female) & 0.295 & 0.438 & 0.453 & 1.34 & $0.57-3.17$ & 0.501 \\
\hline Radiotherapy dose ( $\geq 73.27$ vs $<73.27$ ) & -0.525 & $0.45 \mathrm{I}$ & 1.356 & 0.59 & $0.24-1.43$ & 0.244 \\
\hline AJCC stage (IVa vs III) & -0.529 & 0.438 & 1.459 & 0.59 & $0.25-1.39$ & 0.227 \\
\hline Chemotherapy (no vs yes) & 0.919 & 1.026 & 0.801 & 2.51 & $0.33-18.72$ & 0.371 \\
\hline Targeted therapy (no vs yes) & -0.203 & 0.745 & 0.074 & 0.82 & $0.19-3.52$ & 0.785 \\
\hline Sensitization therapy (no vs yes) & -0.194 & 0.446 & 0.189 & 0.82 & $0.34-1.98$ & 0.664 \\
\hline MAP2K6 (high vs low) & 1.240 & 0.553 & 5.020 & 3.46 & $1.17-10.22$ & 0.025 \\
\hline
\end{tabular}

Abbreviations: AJCC, American Joint Committee on Cancer; NPC, nasopharyngeal carcinoma.

Table 4 Multivariate Cox regression analyses of clinical prognosis in patients with NPC

\begin{tabular}{|c|c|c|c|c|c|c|}
\hline Factors & $\boldsymbol{\beta}$ & SE & Wald $\chi^{2}$ & HR & $95 \% \mathrm{Cl}$ & $P$-value \\
\hline Age ( $\geq 45$ vs 45 years) & -0.226 & 0.444 & 0.358 & 0.77 & $0.32-1.83$ & 0.550 \\
\hline Gender (male vs female) & 0.222 & 0.426 & 0.272 & 1.25 & $0.54-2.88$ & 0.602 \\
\hline Radiotherapy dose ( $\geq 73.27$ vs $<73.27$ ) & -0.655 & 0.458 & 2.043 & 0.52 & $0.21-1.28$ & 0.519 \\
\hline AJCC stage (IVa vs III) & -0.217 & 0.446 & 0.237 & 0.81 & $0.34-1.93$ & 0.626 \\
\hline Chemotherapy (no vs yes) & 1.189 & 1.032 & 1.327 & 3.28 & $0.43-24.84$ & 0.249 \\
\hline Targeted therapy (no vs yes) & 0.118 & 0.643 & 0.034 & 1.13 & $0.32-3.97$ & 0.854 \\
\hline Sensitization therapy (no vs yes) & 0.006 & 0.455 & 0.000 & 1.01 & $0.4 I-2.45$ & 0.990 \\
\hline MAP2K6 (high vs low) & 1.224 & 0.564 & 4.714 & 3.40 & $1.13-10.26$ & 0.030 \\
\hline
\end{tabular}

Abbreviations: AJCC, American Joint Committee on Cancer; NPC, nasopharyngeal carcinoma.

could possibly be a novel diagnostic or prognostic biomarker in these cancers. It was reported that the fusion protein TATOSBP-MAP2K6 (E) can induce the autophagic cell death by activating the P38MAPK signaling pathway, thereby inhibiting the proliferation, invasion, and metastasis of ovarian cancer cisplatin-resistant SKOV3/ DDP cells cisplatin sensitivity. To a certain extent, it can reverse cisplatin resistance. It is suggested that the fusion protein TAT-OSBP-MAP2K6 (E) is expected to be a new chemotherapeutic agent in the treatment of cisplatin in ovarian cancer and provide a new therapeutic mode for the treatment of ovarian cancer. ${ }^{23,24}$ Galan-Moya et $\mathrm{a}^{25}$ reported that the balance between MKK6 and MKK3 mediated $\mathrm{p} 38 / \mathrm{MAPK}$-associated resistance to cisplatin in non-small-cell lung cancer cell lines, and these results were also confirmed in head and neck carcinoma-derived cell line. Studies about the role of MAP2K6 are few in patients with NPC. In fact, we simply compared the expression level of MAP2K6 between normal tissue and NPC tissue using some database from Gene Expression Omnibus (GEO), and no significant difference was observed. However, it was suggested that phosphorylation in the Ser207 and Thre211 can activate the MAP2K under the stress stimulations such as radiation, inflammatory reaction, osmotic shock, and hypoxia. ${ }^{26}$ It is possible that MAP2K6 can be activated by radioactive X-rays.

Protein kinases such as MAP2K6 are important components of MAPK signal pathway. The dual specificity protein kinases consist of the following kinases. MAP3K3 mainly catalyzes the phosphorylation of threonine and tyrosine of MAPK such as p38, MAPK11, and so on, which regulated the cellular responses to inflammation factors and types of stress, while MAP2K6 is the activator of MAPK during the process of response. ${ }^{27}$ MAPK consisted of three types, c-Jun aminoterminal kinase, $\mathrm{p} 38$, and extracellular signal-regulated protein kinase. They can be activated by phosphorylation in Tyr and Ser, which increased the expression of some proliferation gene. Schiemann et $\mathrm{al}^{28}$ first reported that leukemia inhibitory factor (LIF) receptor was linked to MAPK. An obvious dose-response relationship was observed when 3T30L1 (cell extracts) processed by LIF in vitro was bound to the intracellular domain of LIF receptor. The activity of MAPK increased with LIF concentration. This result indicated that MAPK was correlated with LIF phosphorylation. MAPK was activated and combined to the Ser1044 area of intracellular domain of LIF receptor. Further study found that LIFR could be a substrate of MAPK. The phosphorylation of serine location accelerated the procession of itself intracellular phagocytosis. In other words, the activity of MAPK quantitatively regulated the LIF receptor in the surface membranes of cells. Recent study reported that serum LIF was associated with radiosensitivity in NPC patients. ${ }^{29}$ In this study, the author first found that LIF significantly promoted the NPC tumor growth and enhanced radioresistance in vitro and vivo. LIF 
treatment can activate mTORC1/p70S6K signaling pathway and enhanced tumor growth and radioresistance. Treatment with LIF receptor or the mTOR inhibitor rapamycin reversed the LIF-mediated effect. LIF-mediated effect, which is the growth of NPC tumor was in arrest and the sensitivity to irradiation was increased. We assumed that this is the mechanism of MAP2K6-mediated radioresistance in NPC tumor. This speculation gives us an important clue. Of course, further research was required.

Some study limitations should be addressed. The present study has a relatively small sample size, and larger sample size is needed to validate the results. Moreover, the present study did not explore the molecular mechanism, and experiments in vivo and vitro should be performed. Besides the adjusted factors are limited, some potential factors may affect the results. Finally, few patients received operation treatment. The adjacent tissues were unavailable for now. We did not compare the expression levels of MAP2K6 in the tissue. According to the GEO data set (GSE53819 and GSE12452), expression of MAP2K6 cannot be confirmed. But it is reported that MAP2K6 was involved in the signaling pathway of NPC, including stress-induced cell cycle arrest, transcription activation, and apoptosis. This can explain the potential role of MAP2K6 in the NPC radiotherapy.

Taken together, the present results indicated that MAP2K6 was correlated with radioresistance in NPC patients, and the elevated expression levels of MAP2K6 predicted poor prognosis. MAP2K6 may be a valuable biomarker for predicting tumor prognosis and a new therapy target. Future study should focus on the molecular mechanism, especially in the $\mathrm{LIF} / \mathrm{mTOR} / \mathrm{p} 70 \mathrm{~S} 6 \mathrm{~K}$ signal pathway.

\section{Acknowledgment}

This study was supported by the Youth Foundation of Xiangya Hospital Central South University (2018Q012), China Postdoctoral Science Foundation (2017M612597) and National Key Clinical Program (Department of Oncology, Xiangya Hospital Central South).

\section{Disclosure}

The authors report no conflicts of interest in this work.

\section{References}

1. Yu MC, Yuan JM. Epidemiology of nasopharyngeal carcinoma. Semin Cancer Biol. 2002;12(6):421-429.

2. DeNittis AS, Liu L, Rosenthal DI, Machtay M. Nasopharyngeal carcinoma treated with external radiotherapy, brachytherapy, and concurrent/ adjuvant chemotherapy. Am J Clin Oncol. 2002;25(1):93-95.
3. Zhou Q, He Y, Zhao Y, Wang Y, Kuang W, Shen L. A study of 358 cases of locally advanced nasopharyngeal carcinoma receiving intensitymodulated radiation therapy: improving the seventh edition of the American joint committee on cancer T-staging system. Biomed Res Int. 2017;2017(1419676):11.

4. He Y, Zhou Q, Shen L, et al. A retrospective study of the prognostic value of MRI-derived residual tumors at the end of intensity-modulated radiotherapy in 358 patients with locally-advanced nasopharyngeal carcinoma. Radiat Oncol. 2015;10:89.

5. Song T, Liang BL, Huang SQ, Xie BK, Ding ZX, Shen J. [Magnetic resonance imaging manifestations of radiation injury in brain stem and cervical spinal cord of nasopharyngeal carcinoma patients after radiotherapy]. Ai Zheng. 2005;24(3):357-361. Chinese.

6. Sun X, Su S, Chen C, et al. Long-term outcomes of intensity-modulated radiotherapy for 868 patients with nasopharyngeal carcinoma: an analysis of survival and treatment toxicities. Radiother Oncol. 2014;110(3):398-403.

7. Xu P, Min Y, Blanchard $\mathrm{P}$, et al. Incidence of small lymph node metastases in patients with nasopharyngeal carcinoma: Clinical implications for prognosis and treatment. Head Neck. 2017;39(2): 305-310.

8. Chua MLK, Wee JTS, Hui EP, Chan ATC. Nasopharyngeal carcinoma. Lancet. 2016;387(10022):1012-1024.

9. Quan C, Xiao J, Liu L, Duan Q, Yuan P, Zhu F. Protein kinases as tumor biomarkers and therapeutic targets. Curr Pharm Des. 2017;23(29):4209-4225.

10. Cuenda A, Lizcano JM, Lozano J. Editorial: mitogen activated protein kinases. Front Cell Dev Biol. 2017;5:80.

11. Kyriakis JM, Avruch J. Mammalian MAPK signal transduction pathways activated by stress and inflammation: a 10-year update. Physiol Rev. 2012;92(2):689-737.

12. Parray AA, Baba RA, Bhat HF, et al. MKK6 is upregulated in human esophageal, stomach, and colon cancers. Cancer Invest. 2014;32(8): 416-422.

13. Yang S, Chen J, Guo Y, et al. Identification of prognostic biomarkers for response to radiotherapy by DNA microarray in nasopharyngeal carcinoma patients. Int J Oncol. 2012;40(5):1590-1600.

14. Huang X, Liu M, Sun H, et al. HK2 is a radiation resistant and independent negative prognostic factor for patients with locally advanced cervical squamous cell carcinoma. Int J Clin Exp Pathol. 2015;8(4):4054-4063.

15. Guo-Qing P, Yuan Y, Cai-Gao Z, Hongling Y, Gonghua H, Yan T. A study of association between expression of hOGG1, VDAC1, HK-2 and cervical carcinoma. J Exp Clin Cancer Res. 2010;29:129.

16. He YX, Wang Y, Cao PF, et al. Prognostic value and predictive threshold of tumor volume for patients with locally advanced nasopharyngeal carcinoma receiving intensity-modulated radiotherapy. Chin J Cancer. 2016;35(1):96.

17. Zhao Y, Shen L, Huang X, et al. High expression of Ki-67 acts a poor prognosis indicator in locally advanced nasopharyngeal carcinoma. Biochem Biophys Res Commun. 2017;494(1-2):390-396.

18. He Y, Wang Y, Shen L, et al. Prognostic value of the distance between the primary tumor and brainstem in the patients with locally advanced nasopharyngeal carcinoma. BMC Cancer. 2016;16:114.

19. Yoshizawa T, Hammaker D, Boyle DL, et al. Role of MAPK kinase 6 in arthritis: distinct mechanism of action in inflammation and cytokine expression. J Immunol. 2009;183(2):1360-1367.

20. Remy G, Risco AM, Iñesta-Vaquera FA, et al. Differential activation of p38MAPK isoforms by MKK6 and MKK3. Cell Signal. 2010;22(4):660-667.

21. Sturchler E, Feurstein D, McDonald P, Duckett D. Mechanism of oxidative stress-induced ASK1-catalyzed MKK6 phosphorylation. Biochemistry. 2010;49(19):4094-4102.

22. Parray AA, Baba RA, Bhat HF, et al. MKK6 is upregulated in human esophageal, stomach, and colon cancers. Cancer Invest. 2014;32(8):416-422. 
23. Liao H, Kang JL, Jiang WY, Deng C, Yuan J, Shuai R. Delivery of constitutively active mutant MKK6(E) with TAT-OSBP induces apoptosis in human ovarian carcinoma HO8910 cells. Int J Gynecol Cancer. 2015;25(9):1548-1556.

24. Zhong J, Kang J, Wang X, Jiang W, Liao H, Yuan J. TAT-OSBP-1MKK6(E), a novel TAT-fusion protein with high selectivity for human ovarian cancer, exhibits anti-tumor activity. Med Oncol. 2015;32(4):118.

25. Galan-Moya EM, de La Cruz-Morcillo MA, Llanos Valero M, et al. Balance between MKK6 and MKK3 mediates p38 MAPK associated resistance to cisplatin in NSCLC. PLoS One. 2011;6(12):e28406.

26. Humphreys JM, Piala AT, Akella R, He H, Goldsmith EJ. Precisely ordered phosphorylation reactions in the $\mathrm{p} 38$ mitogen-activated protein (MAP) kinase cascade. J Biol Chem. 2013;288(32):23322-23330.
27. Han J, Lee JD, Jiang Y, Li Z, Feng L, Ulevitch RJ. Characterization of the structure and function of a novel MAP kinase kinase (MKK6). $J$ Biol Chem. 1996;271(6):2886-2891.

28. Schiemann WP, Graves LM, Baumann H, et al. Phosphorylation of the human leukemia inhibitory factor (LIF) receptor by mitogenactivated protein kinase and the regulation of LIF receptor function by heterologous receptor activation. Proc Natl Acad Sci U S A. 1995;92(12):5361-5365.

29. Liu SC, Tsang NM, Chiang WC, et al. Leukemia inhibitory factor promotes nasopharyngeal carcinoma progression and radioresistance. J Clin Invest. 2013;123(12):5269-5283.

\section{Publish your work in this journal}

Cancer Management and Research is an international, peer-reviewed open access journal focusing on cancer research and the optimal use of preventative and integrated treatment interventions to achieve improved outcomes, enhanced survival and quality of life for the cancer patient. The manuscript management system is completely online and includes a very quick and fair peer-review system, which is all easy to use. Visit http://www.dovepress.com/testimonials.php to read real quotes from published authors. 